\title{
Incidence, prevalence and epidemiology of spinal cord injury: what learns a worldwide literature survey?
}

\author{
M Wyndaele ${ }^{1}$ and J-J Wyndaele*,1 \\ ${ }^{1}$ Department of Urology, University Hospital Antwerp, Edegem, Belgium
}

\begin{abstract}
Study design: Literature survey.
Objectives: To provide an overview of the literature data on incidence, prevalence and epidemiology of spinal cord injury (SCI) worldwide and to study their evolution since 1977.

Setting: University Antwerp.

Methods: The literature from 1995 onwards was searched on Pubmed. To include evolutionary data, we incorporated the results of three older studies.

Results: Two studies gave prevalence of SCI, and 17 incidence of SCI. The published data on prevalence of SCI was insufficient to consider the range of 223-755 per million inhabitants to be representative for a worldwide estimate. Reported incidence of SCI lies between 10.4 and 83 per million inhabitants per year. One-third of patients with SCI are reported to be tetraplegic and $50 \%$ of patients with SCI to have a complete lesion. The mean age of patients sustaining their injury at is reported as 33 years old, and the sex distribution (men/women) as 3.8/1.

Conclusion: There is a need for improved registration of SCI, and publication of the findings in many parts of the world. This survey pleads for uniformity in methodology. The data show that the reported incidence and prevalence have not changed substantially over the past 30 years. Data from Northern America and Europe show higher figures for incidence, but prevalence figures have remained the same. Epidemiology of SCI seems to have changed during the last decades with a higher percentage of tetraplegia and of complete lesions. If such evolution is present worldwide, how it could eventually be prevented needs to be studied.
\end{abstract}

Sponsorship: Not applicable.

Spinal Cord (2006) 44, 523-529. doi:10.1038/sj.sc.3101893; published online 3 January 2006

Keywords: incidence; prevalence; epidemiology; spinal cord injury

\section{Introduction}

Knowledge of incidence and prevalence of spinal cord injury (SCI) is important both because of their high personal, bio-psychological impact and because of their high socio-economic consequences, short-term as well as long-term. Incidence rates reflect the level of control of SCI and the possible need for improved prevention. On the other hand, prevalence rates have an impact on health care and on social and personal resources.

The last international comparison, from $1995,{ }^{1}$ indicated that there were less prevalence studies than incidence studies. Patients with SCI involuntarily place a heavy burden on the health care system, not only in the phase of acute care, but also in the first years following injury. Illustrations of this are: secondary complications needing hospitalization (eg urinary tract infection and pressure sores); the need for home care services and for

*Correspondence: J-J Wyndaele, Department of Urology, University Hospital Antwerp, Wilrijkstraat 10, B 2650 Edegem, Belgium extra physician contacts; and other health care problems that are more common in people with SCI (eg psychological disorders). ${ }^{2}$ SCI-related cost in the USA was estimated $\$ 9.7$ million per year. ${ }^{3}$

Life expectancy of patients with SCI continues to increase. The median survival time of patients sustaining an SCI between the age of 25 and 34 years has been predicted to be 38 years postinjury, with $43 \%$ surviving for at least 40 years. ${ }^{4}$ These figures suggest an increase in life expectancy of about 5 years over previous research (1983) on the same cohort. ${ }^{4}$

This review has looked into the literature data on SCI incidence and prevalence rates from countries all over the world. It tends to evaluate if an international comparison can be made. It also compares more recent data with those of a previous international comparison by Blumer and Quine. ${ }^{1}$ They estimated SCI incidence to be between 13 and 33 cases per million per year, whereas their estimate of SCI prevalence rates ranged from 110 
to 1120 per million population. Furthermore, this study looks into published data on the evolution of these rates over the last 30 years and into the differences in methodology used for estimation of incidence and prevalence rates. Finally, it compares the current SCI epidemiology to the epidemiology in older studies.

\section{Methods}

The literature was searched on Pubmed, using the search terms: 'Epidemiology of spinal cord injury'; 'Prevalence of spinal cord injury'; and 'Incidence of spinal cord injury'. We limited our search to articles published from the 1st of January 1995 onwards, because data before were included in the Blumer and Quine review. ${ }^{1}$

We screened the found items for articles applicable to this review. To complete our search, we used the 'Related articles' option on every applicable article and screened these lists as well. To include an evolutionary analysis, we used the results of the studies by Kurtzke and by Tricot. ${ }^{6}$

\section{Results}

Our search produced 809 items for the search term 'Epidemiology of SCI', 1123 items for 'Incidence of SCI' and 889 items for 'Prevalence of SCI'. A total of 19 references dealt with our subject in the used period frame and are evaluated here.
Results in the literature are summarised in Table 1 for prevalence of SCI and in Table 2 for incidence of SCI, chronologically listed, by author and year of publication, observation period, country of study and prevalence per million inhabitants and incidence per million inhabitants per year, respectively.

Incidence and prevalence rates found by four reviews of global studies performed over the past 30 years are chronologically listed in Table 3 (global) and in Table 4 (per continent).

If, within the found articles, additional information on the epidemiology of spinal cord injuries was available, it was summarised in Table 5, by author and year of publication, giving percentage of paraplegia, percentage of tetraplegia, percentage of complete lesions, and percentage of incomplete lesions, age, and sex distribution. We listed the two older studies first, followed by a list dependent on available data.

Prevalence of SCI (Table 1)

Only two manuscripts of SCI prevalence were found. One study is based on information on SCI in Australia, and the other one relates to SCI in Helsinki, Finland. Both studies used different methods to measure prevalence.

In the Australian study, ${ }^{7}$ based on the Australian Spinal Cord Injury Register (ASCIR), prevalence was estimated based on the relationship of prevalence $(P)$ to

Table 1 Prevalence of spinal cord injury, studies since 1995

\begin{tabular}{lclr}
\hline & Observation period & Country & Prevalence per million inhabitants \\
\hline O'Connor $^{7}$ & 1997 & Australia & 681 \\
Dahlberg et $^{8}$ & 1999 & Finland: Helsinki & 280 \\
\hline
\end{tabular}

Table 2 Incidence of spinal cord injury, studies since 1995

\begin{tabular}{|c|c|c|c|}
\hline & Observation period & Country & Incidence per million inhabitants per year \\
\hline Karamehmetoglu et al $l^{9}$ & 1992 & Istanbul, Turkey & 21 \\
\hline Warren et $a l^{10}$ & $1991-1993$ & Alaska, USA & 83 \\
\hline Shingu et $a l^{11}$ & $1990-1992$ & Japan & 40.2 \\
\hline Silberstein et $a l^{12}$ & $1989-1993$ & Novosibirsk, Russia & 29.7 \\
\hline Maharaj $^{13}$ & $1985-1994$ & Fiji Islands & 18.7 \\
\hline Chen et $a l^{14}$ & $1992-1996$ & Taiwan & 18.8 \\
\hline Otom et $a l^{15}$ & $1988-1993$ & Jordan & 18 \\
\hline Karamehmetoglu et $a l^{16}$ & 1994 & Rural areas, Turkey & 16.9 \\
\hline Martins et $a l^{17}$ & 1989-1992 & Coimbra, Portugal & 25.4 \\
\hline van Asbeck et al ${ }^{18}$ & 1994 & The Netherlands & 10.4 \\
\hline Karacan et $a l^{19}$ & 1992 & Turkey & 12.7 \\
\hline Surkin et $a l^{20}$ & 1992-1994 & Mississippi, USA & 59 \\
\hline Burke et $a l^{21}$ & & Kentucky, Indiana, USA & 27.1 \\
\hline $\mathrm{O}^{\prime}$ Connor ${ }^{22}$ & 1998-1999 & Australia, (age-standardized) & \\
\hline & & & 14.5 \\
\hline Pickett et $a l^{23}$ & 1994-1999 & Ontario, Canada & From $37.2-46.2$ \\
\hline Dryden et $a l^{24}$ & $1997-2000$ & Alberta, Canada & 44.3 \\
\hline Albert et $a l^{25}$ & 2000 & France & 19.4 \\
\hline
\end{tabular}


Table 3 Evolution of incidence and prevalence of spinal cord injury over 30 years

\begin{tabular}{llcc}
\hline Year of review & Authors & Incidence per million inhabitants per year & Prevalence per million inhabitants \\
\hline 1977 & Kurtzke $^{5}$ & 30 & 520 \\
1981 & Tricot $^{6}$ & 21.7 & Not mentioned \\
$1975-1995$ & Blumer and Quine & $13-71(34.4)$ & $110-1120(554)$ \\
$1995-2005$ & This review & $10.4-83(29.5)$ & $223-755(485)$ \\
\hline
\end{tabular}

()$=$ average of incidence and prevalence

Table 4 Evolution of incidence and prevalence of spinal cord injury in literature over 30 years per continent

\begin{tabular}{|c|c|c|c|c|c|c|c|}
\hline \multirow{2}{*}{ Review } & \multicolumn{2}{|c|}{ Northern America } & \multicolumn{2}{|c|}{ Europe } & \multicolumn{2}{|c|}{ Australia } & \multirow{2}{*}{$\frac{\text { Asia }}{\text { Incidence }}$} \\
\hline & Incidence & Prevalence & Incidence & Prevalence & Incidence & Prevalence & \\
\hline Tricot $^{6}$ & 43.3 & & 13.9 & & 15.8 & & 27.1 \\
\hline Blumer and Quine ${ }^{1}$ & 46 & 681 & 15.5 & 250 & 19 & 370 & \\
\hline This review & 51 & 755 & 19.4 & 252 & 16.8 & 681 & 23.9 \\
\hline
\end{tabular}

Incidence: average number per million inhabitants per year. The Australian incidence number used is the crude rate as opposed to the age-standardized rate in Table 2

Prevalence: average number per million inhabitants

Table 5 Epidemiology of spinal cord injury, literature data

\begin{tabular}{|c|c|c|c|c|c|c|}
\hline & Paraplegia (\%) & Tetraplegia (\%) & Complete (\%) & Incomplete (\%) & Age (years) & Men/women \\
\hline Kurtzke $^{5}$ & 86.40 & 13.60 & 40.00 & 60.00 & $15-34$ & $5.0 / 1$ \\
\hline Tricot $^{6}$ & $42.68-91.3$ & $8.7-57.32$ & & & 38.2 & $4.6 / 1$ \\
\hline van Asbeck et al ${ }^{18}$ & 43.00 & 57.00 & 48.70 & 51.30 & & $3.0 / 1$ \\
\hline Maharaj $^{13}$ & 69.00 & 31.00 & 52.10 & 47.90 & $16-30: 35 \%$ & $4.0 / 1$ \\
\hline Dahlberg et $a l^{8}$ & 54.00 & 46.00 & 43.00 & 57.00 & 31.00 & $3.0 / 1$ \\
\hline Karacan et $a l^{19}$ & 67.80 & 32.18 & & & $35.5 \pm 15.1$ & $2.5 / 1$ \\
\hline Karamehmetoglu et al ${ }^{9}$ & 67.00 & 33.00 & & & 33.00 & $3.0 / 1$ \\
\hline Karamehmetoglu et $a l^{16}$ & 58.70 & 41.30 & & & 31.3 & $5.8 / 1$ \\
\hline Chen et $a l^{14}$ & & & & & 46.1 & $3.0 / 1$ \\
\hline Martins et $a l^{17}$ & & & & & 50.00 & $3.0 / 1$ \\
\hline Surkin et $a l^{20}$ & & & & & & $4.4 / 1$ \\
\hline
\end{tabular}

the multiplicative product of disease incidence $(I)$ and disease duration $(D): P=I \times D .^{7}$ In the Finnish study, cases were identified using SCI registers. ${ }^{8}$

We also found three other reports dealing with the same subject: the Stockholm Spinal Cord Injury Study (SSCIS, 1996) used SCI registers to estimate SCI prevalence and estimated it to be 223 per million inhabitants. ${ }^{26}$ The National Center for Injury Prevention and Control (NCIPC) estimated 200000 inhabitants of the USA to have a SCI in 2001, which converts to a prevalence of about 700 per million population. ${ }^{27}$ The National Spinal Cord Injury Statistical Centre (NSCISC) database, which was recently been positively evaluated, ${ }^{28}$ estimated the number of people in the United States, who are alive and have a SCI, to be approximately 250000 persons in July 2005, with a range of 225000-288000 persons. ${ }^{29}$ This converts to a prevalence of about 755 per million population, with a range of $679-870$ per million population.
Prevalence rates of Stockholm (223/million) and of Helsinki (280/million) are comparable. The same goes for the prevalence rates of Australia (681/million) and of the USA (700-755/million). Unfortunately, we found only these five studies on prevalence of SCI, and all of them are from developed countries. We have not found data on Asia, Africa, South-America, and the rest of Europe and therefore we cannot produce a worldwide SCI prevalence estimate.

Incidence of SCI (Table 2)

In all, 17 studies of SCI incidence were found. To be able to make a comparison with prevalence estimates, we included only incidence studies based on a postinjury acute care and on a rehabilitation population. Most studies (15/17) were retrospective.

Seven of these studies related to SCI in Europe (Turkey, Russia, Portugal, The Netherlands, and 
France) with an incidence variation from 10.4 per million per year to 29.7 per million per year. Five studies were based on information from Northern America (Alaska, Mississippi, Kentucky, Indiana, Ontario, and Alberta), showing an incidence between 27.1 per million per year and 83 per million per year. A report from the NSCISC estimated the annual incidence of SCI, not including those who die at the scene of the accident, to be approximately 40 cases per million population or approximately 11000 new cases each year. ${ }^{29}$ Four studies were done in Asia (Jordan, Japan, Taiwan, and Fiji Islands) with an incidence between 18.0 per million per year and 40.2 per million per year. From Australia, there was one study, estimating the age-standardized SCI incidence at 14.5 per million per year. The crude SCI incidence is 16.8 per million per year. ${ }^{7}$

Again, most studies are from developed countries. No studies from South-America or from Africa were found. The global estimate of SCI incidence from literature lies between 10.4 per million per year and 83 per million per year when only patients that survived before hospital admission were included.

Three studies also contained data including the prehospital mortalities. Martins et $a l^{17}$ estimated this total incidence to be 57.8 per million per year in Portugal, Surkin et $a l^{20}$ estimated it to be 77 per million per year in Mississippi (USA) and Dryden et $a l^{24}$ estimated it to be 52.5 per million per year in Alberta (Canada). This results in a prehospital mortality rate ranging from 15 to $56 \%$.

\section{Evolution of SCI incidence and prevalence over 30 years} (Tables 3 and 4)

Where older studies produced an average of the available data (sum divided by the number), the more recent studies produce a range of data. Which method is preferable will be discussed further on. In order to compare older and recent rates, we calculated the average of the latter (between brackets).

Important is from which regions, the reviews give the data. Tricot reviewed four studies from Europe (RFA, Switzerland, Norway and France), two studies from Australia (Victoria and Brisbane), two studies from Northern America (Northern California) and one study from Asia (Japan). Blumer and Quine reviewed 10 studies from Northern America (Northern California, Minnesota, Canada and Greenland, USA global), two studies from Europe (France and Iceland), one study from Asia (Kashmir) and one study from Australia. Table 4 summarizes the averages of the data found.

\section{Epidemiology of spinal cord injured patients (Table 5)}

Of the 19 studies found on prevalence and incidence of SCI in this search, nine contained additional information about the epidemiology of SCI patients. For comparison, two older studies have been added to Table 5.

\section{Discussion}

In the 10 years after Blumer and Quine's review, only two studies on prevalence of SCI have been published. This lack of data is surprising as knowledge of prevalence of SCI must be considered very important because of the impact of SCI on the health care system. We suspect that more countries must have national SCI registers, and therefore knowledge of prevalence of SCI in their region. To be able to make international comparisons and global estimates possible, such data should be gathered and published.

Both at the time of Blumer and Quine's review and today, there is a lack of information from developing countries where health care is still being developed and where such care is less accessible. ${ }^{30}$ Most available information on prevalence and on incidence of SCI comes from Northern America, Europe and Australia. These three continents made up $20 \%$ of the world population in $1999 .{ }^{30}$ Data on prevalence and incidence of SCI from Asia, Africa and Latin America are necessary to permit a global estimate.

The studies we found used two different methods to measure the prevalence of SCI: case registers and estimates from national registers based on survival times.

Case registers are suitable where the total population is smaller as they require a well-defined population to be reliable. Registers can include new cases of SCI over many years, and therefore they create the opportunity to compare numbers from different time periods and to study evolution. A negative aspect of case registers is that they have to include patients retrospectively at the moment registration starts. As a result of potentially missed patients, prevalence data acquired only from a register are trustworthy many years after registration started. Another negative aspect is that cases living in less accessible areas might be missed. Estimates based on survival times are made on the assumption of the relation between the prevalence and the multiplicative product of incidence and disease duration. This method is suitable for larger populations. A negative aspect of this method, however, is that the final estimate of prevalence can only be made by the product of two other estimates. Inclusion criteria have a big influence on incidence as shown in the results section. ${ }^{17,20,24}$ Cases that died before they got into the hospital, should, strictly taken, be part of an incidence measurement. On the other hand, they should not be part of an incidence estimate to be used in a prevalence calculation. In order to make the multiplication, an estimate of the disease duration applicable to the studied population is needed. Multiple studies report a negative association of older age at injury, higher neurological level and completeness of SCI with survival of patients with SCI. ${ }^{31-36}$ Inclusion of health status, community integration, and economic risk factors may result in higher life expectancy estimates. ${ }^{37}$ All these variables might influence survival duration estimates and thus prevalence calculation by this method, probably making it less accurate. However, 
in larger populations, it is the most realistic method to be used so far.

Table 3 shows that the data on incidence rates as well as on prevalence rates have remained more or less the same over the past 30 years. From this, one might deduct that there are more new cases of SCI, as the population has grown ${ }^{30}$ and that the incidence has not diminished. One might also suppose that the duration of survival remained equal, as incidence is comparable and prevalence has not grown. Comparison between older studies and this review is possible as data relate to the same regions. However, as information from Latin America, Africa and Asia is lacking, global worldwide conclusions are not possible.

Averages of incidence and prevalence were calculated to allow a careful comparison with older studies. We consider the combination of a range and an average to be the best way to publish data on incidence and prevalence. A range clears out the influence of differences (eg demographic) between regions and it clears out the difference in methodology. On the other hand, one study can have a big influence on the results. In our review, most countries gave an incidence of SCI between 15 and 30 per million inhabitants per year, a range of 15 per million per year. As a result of one study with a very low incidence and one study with a very high incidence, the range could, however, become as high as 72 per million per year, a multiplication by 4.8 . The effect of this disadvantage can be diminished by the addition of an average. However, to use an average alone also can give a wrong impression: some regions have an incidence twice the average. Presenting a range as well as an average can help to avoid such underestimation. Therefore, we think that by using both, a more global estimate becomes available as each give a different kind of information and has a different use. They do not clear out each other's limitations, but their associated use permits a better evaluation of the numbers.

Table 4 shows that the averages of data on incidence of SCI in both Northern America and Europe have increased whereas the average of prevalence data has remained the same. By using the disease incidence and duration, an estimate of survival time irrespective of age of injury would be 14.8 years in Northern America, 12.9 years in Europe and 40.5 years in Australia (using the crude incidence and prevalence rates). Compared to the Blumer and Quine review, survival time would seem to have dropped in Europe (16.1 years) and to have remained equal in Northern America (14.8 years). In Australia (19.5 years) on the other hand, survival time would seem to have doubled. Although previous studies have suggested an increase in survival time over the last decades, ${ }^{4}$ data from this review would indicate the opposite. One must be very cautious, however, to get too quick into conclusions taking into account the limitations of the reported numbers and the methodological differences between older and more recent studies. Literature data could indicate that incidence in both Northern America and Europe keep rising whereas prevalence remains more or less the same. A limitation of the calculations is the fact that we use averages. The already described shortcomings and the discrepancy between our calculated estimates and previously published data ${ }^{4}$ illustrates this. The value of an average lies within the number of data used. Our European prevalence average therefore is not accurate and certainly not representative. Only the calculated Australian survival time is near the published estimate. It is unlikely to be coincidental that it is the only estimate not based on averages. If we use the data from the NSCISC to calculate American life expectancy, and herein avoid the use of an average, we get a survival time of 18.9 years, an increase with nearly $28 \%{ }^{29}$ This is still lower than what has been reported by the NSCISC: they estimated the life expectancy of patient injured at 40 years to be 28.3 years in case of paraplegia, 24.4 years in case of low tetraplegia and 21.5 years in case of high tetraplegia. ${ }^{29}$

As conclusion, we can state that to be able to make definite conclusions uniformity in methods and inclusion criteria are mandatory, and many more data are needed.

Table 5 shows that the numbers giving proportion of paraplegia versus tetraplegia have changed compared to older studies. Literature data show that two-thirds of SCI patients are paraplegic, and one-third is tetraplegic whereas in older studies, the proportion of paraplegics used to be up to $90 \%$. A very high percentage of tetraplegics was found in the study from the Netherlands $(57 \%) .{ }^{18} \mathrm{~A}$ demographic study by Jackson et $a l^{38}$ reports a tetraplegia percentage of $54.1 \%$ in the United States. Before 30 years, $40 \%$ of SCI patients had a complete lesion. Recent studies show an increase in complete SCI to $50 \%$.

Except for Portugal and Taiwan, the mean age of patients sustaining their injury at is in their early thirties. This was also reported as such in older studies. The NSCISC fact sheet states that from 1973 to 1979, the average age at injury was 28.7 years and that it has risen to 37.6 years in $2000 .^{29}$

The sex distribution (men/women) of SCI in recent studies is $3.8 / 1$, where it used to be $4.8 / 1$. Men seem to be still more at risk for SCI; however, women do seem to catch up slowly.

We can conclude that most patients with SCI are young men, in the beginning of their thirties, more likely paraplegic, complete or incomplete. This has severe socio-economic consequences. Most of these men will be working to support their family. With a SCI, they will have to rely on help from the health care system, and on the social security system; some of them for the rest of their lives. Others will have to switch jobs, with or without additional training. It is a big change in their own lives, but as they are at the mean productive age, it also influences the economic and social structure of the society they live in.

The increase in percentage of tetraplegics and the increase in percentage of complete lesions is noted in all the available studies. As we reported before, these two factors might, together with older age at injury, have a 
negative influence on survival time. Tetraplegic patients require more care and have great difficulties converting to another job. From this point of view, one can only hope that their percentage as well as the total SCI incidence does not increase more. An Australian study predicted that population growth and aging, and increasing rates of SCI in the elderly will have profound effects on the expected number of SCI patients and their case mix. It predicted a $143 \%$ increase in the number of cases of incomplete tetraplegia, from 88 cases per annum in 1997 to 214 cases per annum in 2021. ${ }^{39}$ Why such evolution occurs, and how it can be prevented eventually, need to be studied further.

\section{Conclusions}

This survey shows the need for improved registration of SCI and the need for publication of the findings in many parts of the world. It pleads for uniformity in methodology, as well in the gathering of information as in the publication of the data, in order to make definite conclusions possible. The studies available show that incidence and prevalence have not changed substantially over the past 30 years, although the incidence of SCI in both Northern America and Europe has risen whereas prevalence remained more or less the same. Epidemiology of SCI has also changed during the last decades: a higher percentage of patients with SCI are tetraplegic and a higher percentage of patients with SCI have a complete lesion. Why such evolution occurred, and how it can be prevented, need to be studied.

\section{References}

1 Blumer CE, Quine S. Prevalence of spinal cord injury: an international comparison. Neuroepidemiology 1995; 14: $258-268$.

2 Dryden DM et al. Utilization of health services following spinal cord injury: a 6-year follow-up study. Spinal Cord 2004; 42: 513-525.

3 Berkowitz M, O'Leary P, Kruse D, Harvey C. Spinal Cord Injury: An Analysis of Medical and Social Costs. Demos Medical Publishing Inc.: New York 1998.

4 McColl MA, Walker J, Stirling P, Wilkins R, Corey P. Expectations of life and health among spinal cord injured adults. Spinal Cord 1997; 35: 818-828.

5 Kurtzke JF. Epidemiology of spinal cord injury. Exp Neurol 1975; 48: 163-236.

6 Tricot A. Etiologie et épidémiologie des lésions médullaires. In: Maury M (ed). La paraplégie 1st edn. Flammarion Médecine-Sciences: Paris 1981 pp 1-8.

7 O'Connor PJ. Prevalence of spinal cord injury in Australia. Spinal Cord 2005; 43: 42-46.

8 Dahlberg A, Kotila M, Leppanen P, Kautiainen H, Alaranta H. Prevalence of spinal cord injury in Helsinki. Spinal Cord 2005; 43: 47-50.

9 Karamehmetoglu SS et al. Traumatic spinal cord injuries in Istanbul, Turkey. An epidemiological study. Paraplegia 1995; 33: 469-471.

10 Warren S, Moore M, Johnson MS. Traumatic head and spinal cord injuries in Alaska (1991-1993). Alaska Med 1995; 37: 11-19.
11 Shingu H, Ohama M, Ikata T, Katoh S, Akatsu T. A nationwide epidemiological survey of spinal cord injuries in Japan from January 1990 to December 1992. Paraplegia 1995; 33: 183-188.

12 Silberstein B, Rabinovich S. Epidemiology of spinal cord injuries in Novosibirsk, Russia. Paraplegia 1995; 33: 322-325.

13 Maharaj JC. Epidemiology of spinal cord paralysis in Fiji: 1985-1994. Spinal Cord 1996; 34: 549-559.

14 Chen HY et al. A nationwide epidemiological study of spinal cord injury in geriatric patients in Taiwan. Neuroepidemiology 1997; 16: 241-247.

15 Otom AS, Doughan AM, Kawar JS, Hattar EZ. Traumatic spinal cord injuries in Jordan - an epidemiological study. Spinal Cord 1997; 35: 253-255.

16 Karamehmetoglu SS et al. Traumatic spinal cord injuries in southeast Turkey: an epidemiological study. Spinal Cord 1997; 35: 531-533.

17 Martins F, Freitas F, Martins L, Dartigues JF, Barat M. Spinal cord injuries epidemiology in Portugal's central region. Spinal Cord 1998; 36: 574-578.

18 van Asbeck FW, Post MW, Pangalila RF. An epidemiological description of spinal cord injuries in The Netherlands in 1994. Spinal Cord 2000; 38: 420-424.

19 Karacan I et al. Traumatic spinal cord injuries in Turkey: a nation-wide epidemiological study. Spinal Cord 2000; 38: 697-701.

20 Surkin J, Gilbert BJ, Harkey rd HL, Sniezek J, Currier M. Spinal cord injury in Mississippi. Findings and evaluation, 1992-1994. Spine 2000; 25: 716-721.

21 Burke DA, Linden RD, Zhang YP, Maiste AC, Shields $\mathrm{CB}$. Incidence rates and populations at risk for spinal cord injury: A regional study. Spinal Cord 2001; 39: 274-278.

22 O'Connor P. Incidence and patterns of spinal cord injury in Australia. Accid Anal Prev 2002; 34: 405-415.

23 Pickett W, Simpson K, Walker J, Brison RJ. Traumatic spinal cord injury in Ontario, Canada. J Trauma 2003; 55: 1070-1076.

24 Dryden DM et al. The epidemiology of traumatic spinal cord injury in Alberta, Canada. Can J Neurol Sci 2003; 30: $113-121$.

25 Albert T, Ravaud JF, Tetrafigap group. Rehabilitation of spinal cord injury in France: a nationwide multicentre study of incidence and regional disparities. Spinal Cord 2005; 43: 357-365.

26 Levi R. The Stockholm spinal cord injury study: medical, economical and psycho-social outcomes in a prevalence population. Doctoral Dissertation 1996, Karolinska Institutet: Stockholm.

27 National Center for Injury Prevention and Control. Injury Fact Book 2001-2002. Centers for Disease Control and Prevention: Atlanta 2001.

28 DeVivo MJ, Go BK, Jackson AB. Overview of the national spinal cord injury statistical center database. J Spinal Cord Med 2002; 25: 335-338.

29 National Spinal Cord Injury Statistical Center. Spinal Cord Injury Facts and Figures at a Glance. Information Sheet June 2005.

30 United Nations. Department of economic and social affairs. Population division. Charting the Progress of Populations. United Nations: New York 2000.

31 Catz A et al. Survival following spinal cord injury in Israel. Spinal Cord 2002; 40: 595-598.

32 DeVivo MJ, Kartus PL, Stover SL, Rutt RD, Fine PR. Seven-year survival following spinal cord injury. Arch Neurol 1987; 44: 872-875. 
33 Frankel HL et al. Long-term survival in spinal cord injury: a fifty year investigation. Spinal Cord 1998; 36: 266-274.

34 Lheritier K, Ravaud JF, Desert JF, Pedelucq JP, O'hanna F, Daures JP. Survival of tetraplegic spinal cord injured persons after the first admission of a rehabilitation center and prognosis factors: a multicenter study of 697 subjects in French centres. Rev Epidemiology Sante Publique 2001; 49: 449-458.

35 Liang HW, Wang YH, Lin YN, Wang JD, Jang Y. Impact of age on the injury pattern and survival of people with cervical cord injuries. Spinal Cord 2001; 39: 375-380.
36 Yeo JD, Walsh J, Rutkowski S, Soden R, Craven M, Middleton J. Mortality following spinal cord injury. Spinal Cord 1998; 36: 329-336.

37 Krause JS, Devivo MJ, Jackson AB. Health status, community integration, and economic risk factors for mortality after spinal cord injury. Arch Phys Med Rehabil 2004; 85: 1764-1773.

38 Jackson AB, Dijkers M, Devivo MJ, Poczatek RB. A demographic profile of new traumatic spinal cord injuries: change and stability over 30 years. Arch Phys Med Rehabil 2004; 85: 1740-1748.

39 O'Connor PJ. Forecasting of spinal cord injury annual case numbers in Australia. Arch Phys Med Rehabil 2005; 86: 48-51. 